Journal of Applied AnALysis

Vol. 8, No. 2 (2002), pp. 297-305

\title{
HUKUHARA'S DERIVATIVE AND CONCAVE ITERATION SEMIGROUPS OF LINEAR SET-VALUED FUNCTIONS
}

\author{
A. SMAJDOR \\ Received August 8, 2000 and, in revised form, October 29, 2001
}

\begin{abstract}
Let $K$ be a closed convex cone with the nonempty interior in a real Banach space and $c c(K)$ denote the family of all nonempty convex compact subsets of $K$. If $\left\{F^{t}: t \geq 0\right\}$ is a concave iteration semigroup of continuous linear set-valued functions $F^{t}: K \rightarrow c c(K)$ with $F^{0}(x)=\{x\}$ for $x \in K$, then

$$
D_{t} F^{t}(x)=F^{t}(G(x))
$$
\end{abstract}

for $x \in K$ and $t \geq 0$, where $D_{t} F^{t}(x)$ denotes the Hukuhara derivative of $F^{t}(x)$ with respect to $t$ and

$$
G(x):=\lim _{s \rightarrow 0+} \frac{F^{s}(x)-x}{s}
$$

for $x \in K$.

1. Let $A$ and $B$ be two subsets of a real vector space $X$. We define the sum of $A$ and $B$ by the formula

$$
A+B=\{a+b: a \in A, b \in B\} .
$$

A subset $K$ of a real vector space is called a cone iff

$$
t K:=\{t x: x \in K\} \subset K
$$

2000 Mathematics Subject Classification. 39B12, 39B52, 26E25.

Key words and phrases. Linear set-valued functions, iterations, Hukuhara's derivative.

ISSN 1425-6908（C) Heldermann Verlag. 
for all positive $t$. A cone is said to be convex iff it is a convex set.

Let $X$ and $Y$ be two real vector spaces and let $K \subset X$ be a convex cone. A set-valued function $F: K \rightarrow n(Y)$, where $n(Y)$ denotes the family of all nonempty subsets of $Y$, is called additive (superadditive) iff

$$
F(x)+F(y)=F(x+y) \quad(F(x)+F(y) \subset F(x+y))
$$

for all $x, y \in K$. A set-valued function $F: K \rightarrow n(K)$ is said to be homogeneous $\left(\mathbb{Q}_{+}\right.$-homogeneous) if $F(\lambda x)=\lambda F(x)$ for all $x \in K$ and $\lambda>0$ $\left(\lambda \in \mathbb{Q}_{+}\right)$, where $\mathbb{Q}_{+}$is the set of all positive rational numbers. A set-valued function $F: K \rightarrow n(K)$ is linear if it is additive and homogeneous.

A set-valued function $F:[0,+\infty) \rightarrow n(Y)$ is said to be concave iff

$$
F(\lambda x+(1-\lambda) y) \subset \lambda F(x)+(1-\lambda) F(y)
$$

for all $x, y \in[0,+\infty)$ and $\lambda \in(0,1)$.

The following property of additive set valued functions is easy to check.

Lemma 1. Let $X$ and $Y$ be two real vector spaces and let $K$ be a convex cone in $X$. Assume that $F: K \rightarrow n(Y)$ is an additive set-valued function and $A, B \in n(K)$. Then

$$
F(A)+F(B)=F(A+B)
$$

where $F(A)=\bigcup\{F(x): x \in A\}$.

We need the following lemma.

Lemma 2 (cf. [7]). Let $A, B$ and $C$ be subsets of a real topological vector space such that

$$
A+B \subset C+B
$$

If $C$ is convex closed and $B$ is non-empty bounded, then

$$
A \subset C \text {. }
$$

Throughout the paper $\mathbb{N}$ denotes the set of all positive integers. All vector spaces are supposed to be real. If $X$ is a topological vector space, then $c(X)$ denotes the set of all compact members of $n(X)$ and $c c(X)$ stands for the set of all convex sets of $c(X)$. By $B(X)$ we denote the set of all bounded members of $n(X)$.

Let $A, B \in c c(X)$. By Lemma 2 there exists at most one set $C \in c c(X)$ fulfilling the equality

$$
A=B+C
$$

M. Hukuhara denoted such set $C$ by $A-B$ and called the difference between $A$ and $B$ (see $[3])$. 
A set valued function $F: X \rightarrow n(Y)$, where $X$ and $Y$ are two topological spaces, is said to be upper semicontinuous (lower semicontinuous) iff the set

$$
F^{+}(U)=\{x \in X: F(x) \subset U\} \quad\left(F^{-}(U)=\{x \in X: F(x) \cap U \neq \emptyset\}\right)
$$

is open for every open subset $U$ of $Y$. A set-valued function is continuous iff it is upper semicontinuous and lower semicontinuous.

Observe that Lemma 1 implies the following.

Lemma 3. Let $X$ and $Y$ be two topological vector spaces and let $K$ be a closed convex cone in $X$. Assume that $F: K \rightarrow c c(K)$ is a continuous additive set-valued function and $A, B \in c c(K)$. If there exists the difference $A-B$, then there exists $F(A)-F(B)$ and $F(A)-F(B)=F(A-B)$.

Let $X$ be a metric space. For $A, A_{n} \in c(X), n \in \mathbb{N}$, the symbol $\lim _{n \rightarrow \infty} A_{n}=A$ means that $\lim _{n \rightarrow \infty} d\left(A_{n}, A\right)=0$, where $d$ denotes the Hausdorff metric derived by the metric in $X$. Let $X$ and $Y$ be two metric spaces. A set-valued function $F: X \rightarrow c(Y)$ is continuous if and only if it is continuous as a single-valued function from $X$ into $c(Y)$ with the Hausdorff metric in $c(Y)$ derived by the metric in $Y$ (see [1], $\S 6$ of Chapter VI).

We will use the following six lemmas.

Lemma 4 (Theorem 3 in [10], see also Lemma 4 in [9]). Let $X$ and $Y$ be two real normed spaces and let $K$ be a convex cone in $X$. Suppose that $\left\{F_{i}: i \in I\right\}$ is a family of superadditive lower semicontinuous in $K$ and $\mathbb{Q}_{+}$-homogeneous set-valued functions $F_{i}: K \rightarrow n(Y)$. If $K$ is of the second category in $K$ and $\bigcup_{i \in I} F_{i}(x) \in B(Y)$ for $x \in K$, then there exists a positive constant $M$ such that

$$
\left\|F_{i}(x)\right\|:=\sup \left\{\|y\|: y \in F_{i}(x)\right\} \leq M\|x\|
$$

for every $i \in I$ and $x \in K$.

Corollary . If $X, Y$ and $K$ are such as in Lemma 4, then the functional

$$
F \mapsto\|F\|:=\sup \left\{\frac{\|F(x)\|}{\|x\|}: x \in K, x \neq 0\right\}
$$

is finite for every $\mathbb{Q}_{+}$-homogeneous superadditive lower semicontinuous setvalued function $F: K \rightarrow B(Y)$.

Lemma 5 (Lemma 5 in [9]). Let $X$ and $Y$ be two real normed spaces and let $d$ be the Hausdorff distance derived from the norm in $Y$. Suppose that $K$ is a convex cone in $X$ with the nonempty interior. Then there exists a positive constant $M_{0}$ such that for every linear continuous set-valued function $F: K \rightarrow c(Y)$ the inequality

$$
d(F(x), F(y)) \leq M_{0}\|F\|\|x-y\|
$$


holds for every $x, y \in K$.

Lemma 6 (Theorem 2 in $[4])$. Let $\left(X, \rho_{X}\right)$ and $\left(Y, \rho_{Y}\right)$ be two metric spaces and let $d_{X}$ and $d_{Y}$ be the Hausdorff metric derived from $\rho_{X}$ and $\rho_{Y}$, respectively. If $F: X \rightarrow n(Y)$ is a set-valued function and $M$ is a positive constant such that

$$
d_{Y}(F(x), F(y)) \leq M \rho_{X}(x, y)
$$

for every $x, y \in X$, then

$$
d_{Y}(F(A), F(B)) \leq M d_{X}(A, B)
$$

for every nonempty subsets $A, B$ of $X$.

Lemma 7 (see e.g. Proposition 2.4.7 in [2]). Let $X$ be a normed space. If $\left(A_{n}\right)$ is a sequence of elements of the set $c(X)$ such that $A_{n+1} \subset A_{n}$ for $n \in \mathbb{N}$, then

$$
\lim _{n \rightarrow \infty} A_{n}=\bigcap_{n=1}^{\infty} A_{n} .
$$

Lemma 8 (Lemma 3 in [8]). Let $K$ be a closed convex cone such that int $K \neq \emptyset$ in Banach space $X$ and let $Y$ be a normed space. If $\left(F_{n}\right)$ is a sequence of continuous additive set-valued functions $F_{n}: K \rightarrow c c(Y)$ such that $F_{n+1}(x) \subset F_{n}(x)$ for all $x \in K$ and $n \in \mathbb{N}$, then the formula

$$
F_{0}(x):=\bigcap_{n=1}^{\infty} F_{n}(x), \quad x \in K
$$

defines a continuous additive set-valued function $F_{0}: K \rightarrow c c(Y)$. Moreover,

$$
\lim _{n \rightarrow \infty} F_{n}(x)=F_{0}(x), \quad x \in K
$$

and the convergence in (1) is uniform on every nonempty compact subset of $K$.

Lemma 9 (Lemma 4 in [8]). Let $D$ be a nonempty set and $Y$ be a normed space. Suppose that $F_{0}, F_{n}: D \rightarrow c(Y)$ are set-valued functions. If the sequence $\left(F_{n}\right)$ uniformly converges to $F_{0}$ on $D$, then

$$
\lim _{n \rightarrow \infty} F_{n}(D)=F_{0}(D) \text {. }
$$

Let $X$ be a normed space and let $\phi:[0, \infty) \rightarrow c c(X)$ be a set-valued function such that the Hukuhara differences $\phi(t+s)-\phi(t)$ exist for nonnegative $t$ and $s$ and the Hukuhara diferences $\phi(t)-\phi(t-s)$ exist for positive $t$ and 
$s \in(0, t)$. Let $t>0$. The Hukuhara derivative of $\phi$ at $t$ is defined by the formula

$$
D \phi(t)=\lim _{s \rightarrow 0+} \frac{\phi(t+s)-\phi(t)}{s}=\lim _{s \rightarrow 0+} \frac{\phi(t)-\phi(t-s)}{s}
$$

whenever both these limits exist (see [3]). Moreover,

$$
D \phi(0)=\lim _{s \rightarrow 0+} \frac{\phi(s)-\phi(0)}{s} .
$$

2. Let $K$ be a nonempty set. A family $\left\{F^{t}: t \geq 0\right\}$ of set-valued functions $F^{t}: K \rightarrow n(K)$ is said to be an iteration semigroup iff

$$
F^{t} \circ F^{s}(x):=F^{t}\left[F^{s}(x)\right]=F^{s+t}(x)
$$

for all $x \in K$ and $t, s \geq 0$.

Let $K$ be a convex cone in a normed space. An iteration semigroup $\left\{F^{t}: t \geq 0\right\}$ of set-valued functions $F^{t}: K \rightarrow c c(K)$ is said to be differentiable iff all set-valued functions $t \mapsto F^{t}(x),(x \in K)$ have Hukuhara's derivative on $[0,+\infty)$. An iteration semigroup $\left\{F^{t}: t \geq 0\right\}$ of set-valued functions $F^{t}: K \rightarrow n(K)$ is said to be concave iff the set-valued function $t \mapsto F^{t}(x)$ is concave for every $x \in K$.

Concave iteration semigroups $\left\{F^{t}: t \geq 0\right\}$ of set-valued functions $F^{t}: K \rightarrow$ $c c(K)$ was introduced in the paper [5] in which also the following lemma was proved.

Lemma 10. Assume that $K$ is a closed convex cone with the nonempty interior in a real Banach space $X$. Let $\left\{F^{t}: t \geq 0\right\}$ be a concave iteration semigroup of continuous linear set-valued functions $F^{t}: K \rightarrow c(K)$ with $F^{0}(x)=\{x\}$ for $x \in K$. Then there exists a set-valued function $G: K \rightarrow$ $c c(K)$ such that the family $\left\{(1 / t)\left(F^{t}-F^{0}\right): t>0\right\}$ uniformly converges to $G$ on every compact subset of $K$, when $t$ tends to zero. Moreover, $G$ is linear continuous and

$$
G(x)=\bigcap_{t>0} \frac{A^{t}(x)-x}{t}
$$

for every $x \in K$.

Some examples of concave iteration semigroups of continuous linear setvalued functions can be found in [5]. We add the following two.

Example 1. Let $F^{t}:[0,+\infty)^{2} \rightarrow c c\left([0,+\infty)^{2}\right)$ for $t \geq 0$ be set-valued functions defined by

$$
F^{t}((x, y))=[x, x \cdot \cosh t+y \cdot \sinh t] \times[y, x \cdot \sinh t+y \cdot \cosh t]
$$


for $(x, y) \in[0,+\infty)^{2}$. Then the family $\left\{F^{t}: t \geq 0\right\}$ of set-valued functions $F^{t}$ is a concave iteration semigroup of continuous linear set-valued functions. Moreover, $G((x, y))=[0, y] \times[0, x]$ for $(x, y) \in[0,+\infty)^{2}$.

Example 2. Let $X$ be the linear space of all functions $f: \mathbb{R} \rightarrow \mathbb{R}$ of the form $f(x)=a x+b$, where $a, b \in \mathbb{R}$. The space $X$ is a Banach space with the norm

$$
\|f\|=|b|+|a|
$$

Let

$$
K:=\{f \in X: a \geq 0\}
$$

The set $K$ is a closed convex cone in $X$ and $f_{0} \in \operatorname{int} K$, where $f_{0}(x)=x+1$ for $x \in \mathbb{R}$. Now, we define set-valued functions $F^{t}$ as follows

$$
F^{t}(f):=\left\{g \in X: \exists_{u \in[0, t]} \forall_{x \in \mathbb{R}} g(x)=f(x+u)\right\}
$$

for $f \in K$. It is easy to check that sets $F^{t}(f)$ are nonempty, compact and convex subsets of $K$. Moreover $F^{t}$ are linear continuous multifunctions and the family $\left\{F^{t}: t \geq 0\right\}$ is a concave iteration semigroup with $G(f)=\{g \in$ $X: g(x) \equiv d, 0 \leq d \leq a\}$.

Lemma 11. Assume that $K$ is a closed convex cone with nonempty interior in a real Banach space $X$. Let $\left\{F^{t}: t \geq 0\right\}$ be a concave iteration semigroup of continuous linear set-valued functions $F^{t}: K \rightarrow c(K)$ with $F^{0}(x)=\{x\}$ for $x \in K$. Then $F^{t}(y)$ converges to $y$ uniformly on every nonempty compact subset $C$ of $K$, when $t \rightarrow 0$.

Proof. Fix $\varepsilon>0$ and a set $C \in c(K)$. According to Lemma 10 there exists $\delta \in(0, \varepsilon /(1+\|G(C)\|))$ such that

$$
d\left(\frac{F^{s}(y)-y}{s}, G(y)\right)<1
$$

for every $y \in C$ and $s \in(0, \delta)$. This implies that

$$
\frac{F^{s}(y)-y}{s} \subset G(y)+S,
$$

where $S$ is the closed unit ball, and

$$
G(y) \subset \frac{F^{s}(y)-y}{s}+S
$$

for $y \in C$ and $s \in(0, \delta)$. Therefore

$$
F^{s}(y) \subset[y+s G(y)]+s S
$$

and

$$
[s G(y)+y] \subset F^{s}(y)+s S
$$


for the same $y$ and $s$. Thus

$$
d\left(F^{s}(y), s G(y)+y\right) \leq s
$$

for $y \in C$ and $0<s<\delta$. Hence we have for the same $y$ and $s$

$$
\begin{aligned}
d\left(F^{s}(y),\{y\}\right) & \leq d\left(F^{s}(y), y+s G(y)\right)+d(y+s G(y),\{y\}) \\
& =d\left(F^{s}(y), y+s G(y)\right)+s\|G(y)\|<s+s\|G(C)\| \\
& =s(1+\|G(C)\|)<\varepsilon .
\end{aligned}
$$

This completes the proof.

Under assumptions of Lemma 10,

$$
G(x):=\lim _{t \rightarrow 0+} \frac{F^{t}(x)-x}{t}
$$

for $x \in K$. Therefore $G$ is the infinitesimal generator of semigroup $\left\{F^{t}: t \geq\right.$ $0\}$ and its domain $D(G)$ is equal to $K$. It is well known that if $\left\{f^{t}: t \geq 0\right\}$ is a strongly continuous semigroup of bounded linear operators on $X$ and $g$ is its infinitesimal generator, then the function $t \mapsto f^{t}(x)$ is differentiable for every $x \in D(g)$ and the equality

$$
\frac{d}{d t} f^{t}(x)=f^{t}(g(x)), x \in D(g)
$$

holds true (see [6]). A similar result for concave iteration semigroup of linear continuous set-valued functions is contained in the following theorem.

Theorem . Let $X$ be a Banach space and let $K$ be a closed convex cone with the nonempty interior. Suppose that $\left\{F^{t}: t \geq 0\right\}$ is a concave iteration semigroup of linear continuous set-valued functions $F^{t}: K \rightarrow c c(K)$ with $F^{0}(x)=\{x\}$. Then this iteration semigroup is differentiable and

$$
D_{t} F^{t}(x)=F^{t}(G(x))
$$

for $x \in K, t \geq 0$, where $D_{t}$ denotes the Hukuhara derivative of $F^{t}(x)$ with respect to $t$ and $G$ is given by Lemma 10 .

Proof. It is obvious that there exist differences

$$
F^{s}(x)-x
$$

for $s>0$ and $x \in K$ so according to Lemma 3 there exist differences

$$
F^{t+s}(x)-F^{t}(x)=F^{t}\left[F^{s}(x)\right]-F^{t}(x)=F^{t}\left(F^{s}(x)-x\right)
$$

and

$$
F^{t}(x)-F^{t-s}(x)=F^{t-s}\left[F^{s}(x)\right]-F^{t-s}(x)=F^{t-s}\left(F^{s}(x)-x\right)
$$

whenever $t>0, s \in(0, t)$ and $x \in K$. 
Lemmas 5 and 6 imply that

$$
\begin{aligned}
& d\left(\frac{F^{t+s}(x)-F^{t}(x)}{s}, F^{t}(G(x))\right)=d\left(F^{t}\left(\frac{F^{s}(x)-x}{s}\right), F^{t}(G(x))\right) \\
& \leq M_{0}\left\|F^{t}\right\| d\left(\frac{F^{s}(x)-x}{s}, G(x)\right)
\end{aligned}
$$

for $x \in K, t>0, s \in(0, t)$. Therefore, in view of Lemma 10

$$
\lim _{s \rightarrow 0+} \frac{F^{t+s}(x)-F^{t}(x)}{s}=F^{t}(G(x))
$$

for $t>0$ and $x \in K$.

Similarly we have

$$
\begin{aligned}
& d\left(\frac{F^{t}(x)-F^{t-s}(x)}{s}, F^{t}(G(x))\right)=d\left(F^{t-s}\left(\frac{F^{s}(x)-x}{s}\right), F^{t-s}\left(F^{s}(G(x))\right)\right) \\
& \leq M_{0}\left\|F^{t-s}\right\| d\left(\frac{F^{s}(x)-x}{s}, F^{s}(G(x))\right)
\end{aligned}
$$

for $t>0, s \in(0, t)$ and $x \in K$.

Fix $x \in K$ and $t>0$. Since $F^{t}(x) \in c(K)$ and

$$
\begin{aligned}
& \left\|F^{t-s}(x)\right\| \leq\left\|\frac{t-s}{t} F^{t}(x)+\frac{s}{t}\{x\}\right\| \\
& \leq \frac{t-s}{t}\left\|F^{t}(x)\right\|+\frac{s}{t}\|x\| \leq \max \left\{\left\|F^{t}(c)\right\|,\|x\|\right\}<\infty .
\end{aligned}
$$

Thus the set $\bigcup_{0 \leq s \leq t} F^{t-s}(x)$ is bounded. By Lemma 4 there exists a positive constant $M$ such that

$$
\left\|F^{t-s}\right\| \leq M
$$

for $s \in[0, t]$. According to (2) and (3) we have

$$
\begin{aligned}
& d\left(\frac{F^{t}(x)-F^{t-s}(x)}{s}, F^{t}(G(x))\right) \leq M_{0} M d\left(\frac{F^{s}(x)-x}{s}, F^{s}(G(x))\right) \\
& \leq M_{0} M d\left(\frac{F^{s}(x)-x}{s}, G(x)\right)+d\left(G(x), F^{s}(G(x))\right) .
\end{aligned}
$$

According to Lemmas 10, 11 and 9, the right part of the last inequality has the limit zero when $s \rightarrow 0+$. Thus

$$
D_{t} F^{t}(x)=F^{t}(G(x)) .
$$

This ends the proof. 


\title{
References
}

[1] Berge, C., Topological Spaces, Oliver and Boyd, Edinburgh, London, 1963.

[2] Edgar, G. A., Measure, Topology and Fractal Geometry, Springer-Verlag, New York, 1990.

[3] Hukuhara, M., Intégration des applications measurables dont la valeur est un compact convexe, Funkcial. Ekvac. 10 (1967), 205-223.

[4] Nadler, Jr., S. B., Multi-valued contraction mappings, Pacific J. Math. 30 (1969), 475-488.

[5] Olko, J., Concave iteration semigroups of linear set-valued functions, Ann. Polon. Math. 71 (1999), 31-38.

[6] Pazy, A., Semigroups of Linear Operators and Applications to Partial Differential Equations, Springer-Verlag, Berlin, 1983.

[7] Rådström, H., An embedding theorem for space of convex sets, Proc. Amer. Math. Soc. 3 (1952), 165-169.

[8] Smajdor, A., Increasing iteration semigroups of Jensen set-valued functions, Aequationes Math. 56 (1998), 131-142.

[9] Smajdor, A., On regular multivalued cosine functions, Ann. Math. Sil. 13 (1999), 271-280.

[10] Smajdor, W., Superadditive set-valued functions and Banach-Steinhaus theorem, Rad. Mat. 3 (1987), 203-214.

\author{
AndRzej SMAJdor \\ PEDAGOGICAL University \\ PODCHORA̧ŻYCH 2 \\ 30-084 KRAKÓW \\ POLAND
}

